\title{
Students' understanding of the structure of deductive proof
}

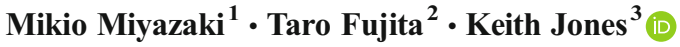

Published online: 16 September 2016

(C) The Author(s) 2016. This article is published with open access at Springerlink.com

\begin{abstract}
While proof is central to mathematics, difficulties in the teaching and learning of proof are well-recognised internationally. Within the research literature, a number of theoretical frameworks relating to the teaching of different aspects of proof and proving are evident. In our work, we are focusing on secondary school students learning the structure of deductive proofs and, in this paper, we propose a theoretical framework based on this aspect of proof education. In our framework, we capture students' understanding of the structure of deductive proofs in terms of three levels of increasing sophistication: Prestructural, Partial-structural, and Holistic-structural, with the Partial-structural level further divided into two sub-levels: Elemental and Relational. In this paper, we apply the framework to data from our classroom research in which secondary school students (aged 14) tackled a series of lessons that provided an introduction to proof problems involving congruent triangles. Using data from the transcribed lessons, we focus in particular on students who displayed the tendency to accept a proof that contained logical circularity. From the perspective of our framework, we illustrate what we argue are two independent aspects of Relational understanding of the Partial-structural level, those of universal instantiation and hypothetical syllogism, and contend that accepting logical circularity can be an indicator of lack of understanding of syllogism. These findings can inform how teaching approaches might be improved so that students develop a more secure understanding of deductive proofs and proving in geometry.
\end{abstract}

Mikio Miyazaki

mmiyaza@shinshu-u.ac.jp

Taro Fujita

t.fujita@exeter.ac.uk

Keith Jones

d.k.jones@soton.ac.uk

1 Institute of Education, Shinshu University, Roku-Ro Nishinagano, Nagano-Shi 380-8544, Japan

2 Graduate School of Education, University of Exeter, Exeter, UK

3 School of Education, University of Southampton, Southampton, UK 
Keywords Proof $\cdot$ Understanding $\cdot$ Structure $\cdot$ Universal instantiation $\cdot$ Hypothetical syllogism

\section{Introduction}

The teaching and learning of proof is recognised internationally as a key component of mathematics education and thus of mathematics curricula (Hanna \& de Villiers, 2008, 2012). Yet it remains the case that students at the secondary school level (and beyond) experience difficulties in understanding proof in mathematics in general, and in geometry in particular. For example, research suggests that when students are asked to prove a mathematical statement there is a tendency for them to do one or more of the following: rely overly on empirical data or concrete examples (e.g., Harel \& Sowder, 2007; Küchemann \& Hoyles, 2006; Martinez \& Pedemonte, 2014); not know where to start as the problem statement may be unfamiliar (e.g., Hoyles \& Healy, 2007); or be unable to use existing knowledge strategically (e.g., Weber, 2001). It seems that even if students might be able to construct a correct proof, they may still verify its correctness via empirical data (e.g., Harel \& Sowder, 2007), and when asked to produce a proof they tend to construct an empirical-numeric proof (Stylianou, Blanton, \& Rotou, 2015). Paralleling this evidence is research reporting that students at school, or even at undergraduate level, show difficulties when they try to read proofs that have been provided for them (e.g., Inglis \& Alcock, 2012; Lin \& Yang, 2007), when they are asked to judge the suitability of a proof (e.g., Hoyles \& Healy, 2007), and when they are asked to write deductive proofs (e.g., McCrone \& Martin, 2004; Senk, 1989).

Here we take deductive proof to be "an appropriate argument supported by valid reasoning" (Hanna \& de Villiers, 2008, p. 329). Such a definition, of course, raises questions about what is an appropriate argument and what constitutes valid reasoning. As Hanna and de Villiers (2012, p. 3) explain, "a narrow view of proof [as solely a formal derivation] neither reflects mathematical practice nor offers the greatest opportunities for promoting mathematical understanding". In fact, in school geometry, proofs are often presented in a somewhat formalised form of "two-column format". Yet, as Herbst and Brach (2006) show, such an approach does not necessarily support students through the creative reasoning processes that they need if they are to be able to build up reasoned arguments for themselves. On the contrary, teaching might usefully include a focus on the structural characteristics of deductive reasoning because these characteristics become increasingly important as students develop from elementary school mathematics to secondary school mathematics and beyond.

In this paper, we focus on the development of secondary school student's understanding of the structure of deductive proofs in geometry. We do this because studies indicate that, in addition to other aspects of proof, students need to understand the structure of deductive proofs if they are to gain an understanding of, and a capability with, how to construct proofs (Durand-Guerrier, Boero, Douek, Epp, \& Tanguay, 2012). As we illustrate later in the paper, our classroom studies are with students of about 14 years of age as this is likely to be the time when they might just be starting to learn to construct proofs in geometry. This means that they are just at the start of learning about the structure of relatively simple proofs, but at the same time are at a stage of learning when many students find proofs difficult to learn. The goal of this paper is to propose a theoretical framework to capture students' understanding of the structure of deductive proofs in secondary school based on our research on students' developing understanding of the structure of deductive proofs. A further goal is to gain insights into how teaching 
approaches might be improved so that students can develop a more secure understanding of proofs and proving.

In this paper, we concentrate on deductive proof in geometry where students are first introduced to ideas of proof and proving. We mainly focus on two issues: why some students cannot find proper reasons or theorems to deduce intermediate statements or a conclusion, and why they might accept a proof that contains logical circularity.

This latter issue has not been fully scrutinised in existing research studies. For example, in a comprehensive consideration of the key questions for mathematics education research on the teaching and learning of proof and proving, Hanna and de Villiers (2008, p. 333) raise the issue of the extent to which students are competent in identifying circular arguments in proofs. Rips (2002) has argued that the psychological study of reasoning should have a natural interest in patterns of thought like circular reasoning, since such reasoning may indicate fundamental difficulties that people may have in constructing and in interpreting even everyday discourse. However, Rips claims that up until his study published in 2002 there appeared to be no prior empirical research on circular reasoning. While Rips reports on a study of young adults, later research by Baum, Danovitch, and Keil (2008) with younger students indicates that by 5 or 6 years of age children show a preference for non-circular explanations and that this appears to become robust by the time youngsters are about 10 years of age. Our intention is to contribute to this matter in the context of the teaching and learning of geometry in secondary school.

In what follows, we begin by outlining what we consider as the structural aspects of deductive proof; in particular, the relationships between singular and universal propositions (see Section 3). In so doing, we explain the necessity for students to understand the structure of proofs. This leads to us proposing a theoretical framework to capture students' developing understanding of the structure of the proofs. Finally, we utilise this framework to analyse qualitatively some selected episodes from our classroom studies of geometry lessons in secondary school in Japan in which students discussed the validity of a proof that contained logical circularity.

\section{The role of students' understanding the structure of deductive proofs}

In focusing on students who are beginning to develop their understanding of the structure of deductive proofs, we start by considering three crucial aspects of proof and proving. The first aspect relates to understanding a proof as a structural object (Miyazaki \& Yumoto, 2009). The second aspect is seeing a proof as an intellectual activity (Balacheff, 1987; Harel \& Sowder, 2007). The third aspect entails focusing on the roles, functions, and meanings of proof and proving (de Villiers, 1990; Hanna \& Jahnke, 1993).

In relation to the first aspect, Duval (2002) proposes that, in order to be able to construct a proof, it is essential to organise premises, conclusions, and theorems. In particular, Duval argues that one of the awareness that learners need to develop entails "becoming aware of the discrepancy between a valid reasoning and a non-valid reasoning" (Duval, 2002, p. 63). Thus, as well as being an appropriate argument supported by valid reasoning, we also take a deductive proof to consist of the following components: singular propositions (premises, conclusions, and intermediate propositions between them), universal propositions (theorems, definitions, etc.), and the appropriate connectives between singular propositions and universal propositions.

Heinze, Cheng, Ufer, Lin, and Reiss (2008, p. 445) argue that constructing a proof with valid reasoning in geometry entails a bridging process involving the following elements: 
understanding "the given information and the status of this information"; recognising "the crucial elements (premise, argument, conclusion)"; constructing, especially in multi-step proofs, "intermediary conditions for the next step of deduction by hypothetical bridging"; and coordinating "the whole process" by organising "the discourse into an acceptable sequence". Within the bridging process, from a predicate logic point of view, at least two types of deductive reasoning are employed: universal instantiation (which deduces a singular proposition from a universal proposition) and hypothetical syllogism (which connects singular propositions logically to bridge between the premises, the intermediate propositions and the conclusion. Here we take hypothetical syllogism as a type of syllogism whose premises belong to not less than one compound proposition including conditionals "if, then"). In particular, in the bridging process, these two types of deductive reasoning are intertwined like the warp and weft of a textile to give legitimate reasons why the conclusion is a logical consequence of the premises (see also Weber \& Alcock, 2005, p. 35, in which they discuss the importance of "warranted implications").

As we have just explained, in our research, we consider the structure of deductive proofs as the network of singular and universal propositions between premises and conclusions, connected with universal instantiation and hypothetical syllogism. As an example, consider a deductive proof of the statement "if $\mathrm{AB}=\mathrm{AC}$ in $\triangle \mathrm{ABC}$, then $\angle \mathrm{ABD}=\angle \mathrm{ACD}$ " that uses the angle bisector $\mathrm{AD}$ (see Fig. 1). Given that "any mathematical theorem is characterised by a statement and a proof and that the relationship between statement and proof makes sense within a particular theoretical context, i.e., a system of shared principles and inference rules" (Mariotti, 2000, p. 29), in this example the two inference rules (universal instantiation and hypothetical syllogism) occur within Euclid's parallel line axiom. Thence, as shown in Fig. 1, the statement "if $\mathrm{AB}=\mathrm{AC}$ in $\triangle \mathrm{ABC}$, then $\angle \mathrm{ABD}=\angle \mathrm{ACD}$ " can be proved.

In the proof in Fig. 1, the reasoning encompasses two singular propositions being deduced by use of universal instantiation, and then the two propositions being connected through use of hypothetical syllogism - all of which makes the reasoning valid (Duval, 2002).

With such examples in mind, we define the "structure of deductive proofs" as the relational network via deductive reasoning that combines singular and universal propositions. Seeing a proof as an object enables appreciation of the components of a proof and their inter-connections, how a proof is composed of these components, and why a proof needs the structure that it has.

The second aspect of proof and proving is seeing a proof as an intellectual activity (Balacheff, 1987; Harel \& Sowder, 2007). By focusing on the activity aspect of proving, it is possible to consider questions of what is involved in the proving activity and what supports it. The third aspect of proof and proving involves the roles, functions, and meanings of proof and proving (de Villiers, 1990; Hanna \& Jahnke, 1993). Examining the functional aspects of proof and proving can contribute to clarifying how proving contributes to understanding what mathematical knowledge is, why it is necessary, and how the functions of proof get embodied with meaning.

These three aspects of proof and proving can be thought of as underpinning the curriculum for the teaching of proof and proving across primary and secondary education (and beyond). What is more, the three aspects need to be interrelated to establish the effective teaching and learning of proof and proving over the long term. In this paper, we particularly focus on the first aspect, the structure of deductive proofs. At the beginning stages of proof teaching, mathematical reasoning is learnt informally; for example, through activities that involve learners in explaining their reasoning inductively. In order to make such informal proving 


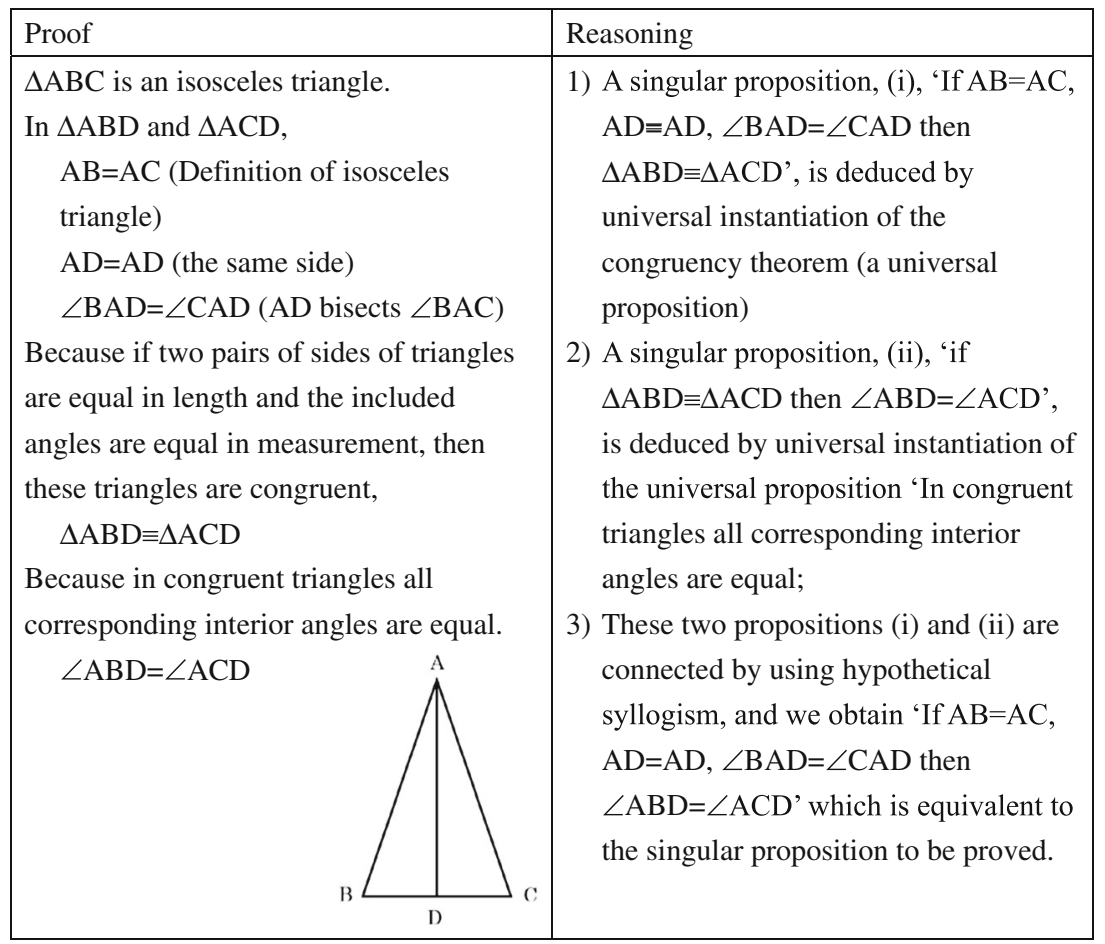

Fig. 1 Proof and reasoning that "the base angles of isosceles triangles are equal"

more formal, students learn that a deductive proof is a deductive method that draws a conclusion from given premises and also how definitions and theorems (i.e. already-proved statements) are used in such proving. Here, a focus on the structure of deductive proofs is crucial. The explicit teaching of the structure of the proofs, in turn, enhances the quality of the teaching and learning of the proofs. All this underlines that the teaching and learning of a proof as a structural object plays a central role in the long-term learning of proof and proving.

\section{Theoretical framework: three levels of understanding of the structure of deductive proofs}

In this section, we examine critically the ideas attained by previous studies based on the structure of deductive proofs in the developmental process of understanding of proofs. From this we propose our framework of three levels of understanding of the structure of deductive proofs.

\subsection{Developmental process of understanding of proofs}

According to Pegg and Tall (2010, p. 469), current frameworks describing conceptual growth can be separated into "global frameworks of long-term growth" such as the van Hiele's levels of geometric thought (1959/1984) and "local frameworks of conceptual growth" such as procept theory (Gray \& Tall, 1994). For example, the van Hiele model, a well-known model of the long-term growth of thinking in geometry, characterises levels of geometric thinking. 
Using this model, existing studies have shown that students develop their understanding of deductive reasoning and proofs in geometry in the latter stages of their thinking, around van Hiele Level 3 and 4 (e.g., Senk, 1989; Usiskin, 1982).

With theories of local developmental processes, Pegg and Tall (2010) suggest, learners start by recognising elements of a concept, then relationships between them, and finally they understand the concept as a whole. In proof and proving, a model that reflects a local developmental perspective is the Reading Comprehension of Geometry Proofs (RCGP) model (Lin \& Yang, 2007; Yang \& Lin, 2008). This model hypothesises four levels of reading comprehension of geometric proofs; these levels are "comprehension of surface (epistemic value)", "comprehension of recognising elements (micro level, logical value)", "comprehension of chaining elements (local level, logical value)", and "comprehension of encapsulation (global level, logical and epistemic values)" (Yang \& Lin, 2008, p. 63).

The RCGP model provides useful insights into how reading comprehension of geometric proofs might progress, but several points remain to be investigated. First, the model is for "reading comprehension of proofs", but in school mathematics, the constructing of proofs is an important reciprocal activity accompanying "reading proofs". Both constructing and reading proofs are regulated by the understandings of proof that contribute to developing related learning progressions (Empson, 2011). Second, as described in Section 2, universal instantiation and hypothetical syllogism are two types of deductive reasoning in the structure of deductive proofs that function to chain the elements of proof but the RCGP, understandably, does not pay detailed attention to the level of "chaining elements". Paying attention to universal instantiation and hypothetical syllogism helps, we argue, to clarify better the "comprehension of chaining elements" and provide a clearer image of "comprehension of encapsulation".

By refining and adjusting the ideas in the RCGP for our research focus, our framework proposes a progression of understanding from recognising individual elements of a deductive proof to recognising the relationships between the elements. In our model, students first pay attention to the components of the proof (the singular and universal propositions to be used), next the inter-relationships between these components, and then gradually they grasp the relational network of the structure of simple proofs (i.e., proofs suitable for secondary school). Our ideas are derived not only from theoretical reflections on the structure of deductive proofs but also empirical insights gained from the accumulation of Japanese research findings on the difficulties of learning and teaching of proofs (MEXT, 2014; Miyazaki \& Fujita, 2015). In the next section we outline our framework in more detail.

\subsection{Three levels of understanding of the structure of deductive proofs}

In our model, the "Pre-structural" level has the most primitive status in terms of understanding the structure of deductive proofs. At this level, students regard a proof as a kind of "cluster" of symbolic objects empty of meaning. As such, when they construct a proof, they cannot see within the structure of the proof that singular propositions are those that are universally instantiated from universal propositions, nor that hypothetical syllogism is necessary to connect singular propositions, and so on (they would not, of course, utilise such terminology). When students at this level are asked, for example, a question involving universal propositions such as "in order to prove that the base angles of an isosceles triangle $\mathrm{ABC}$ are equal, what theorems are needed in order to deduce $\triangle \mathrm{ABD} \equiv \triangle \mathrm{ACD}$ ?" (where $\mathrm{D}$ is the mid-point of $\mathrm{BC}$, see Fig. 1), they would not understand what they were being asked, or they may simply answer with a random singular proposition " $\mathrm{BD}=\mathrm{CD}$ ?". 
Once a student has started paying attention to each component, then in our framework we consider them to be at the Partial-structural Elemental sub-level. This level has echoes of the "Recognizing elements" level of the RCGP (Yang \& Lin, 2008) and the first processes of Heinze et al. (2008)). However, just recognising elements of proofs is not enough to construct valid proofs; a student at this level still needs to recognise some logical chaining relationships between the components of a proof from premises to conclusions (c.f. Yang and Lin, 2008, p. 63; the third process of Heinze et al., 2008).

In order to understand the chaining relationship, first it is necessary to distinguish premises from conclusion. This is because the students need to identify the premise as the starting point (Fawcett, 1938). Further, as we argued above, two types of reasoning (universal instantiations and hypothetical syllogism) construct the chaining relationship. Once students have started paying attention to these two types of reasoning, then we consider them to be at the Partial-structural Relational sub-level. For example, if a student understands universal instantiation (without knowing the terminology), then, when faced with a question such as "In $\triangle \mathrm{ABD}$ and $\triangle \mathrm{ACD}$ (Fig. 1), with $\mathrm{AB}=\mathrm{AC}$ already assumed, what additional premises should be made to prove $\triangle \mathrm{ABD} \equiv \triangle \mathrm{ACD}$ ?", the student can answer by stating, for instance, something akin to "In order to use the condition of congruent triangles, $\angle \mathrm{BAD}=\angle \mathrm{CAD}$ and $\angle \mathrm{ABD}=\angle \mathrm{ACD}$ are needed."

The understandings concerning universal instantiations and hypothetical syllogism are independent; the former is related to seeking grounds for reasoning in proof steps and the latter connects several steps of deductive reasoning. In other words, at the Partial-structural Relational sub-level, corresponding to the "comprehension of chaining elements" level of the RCGP (Yang \& Lin, 2008) and the "bridging process" of Heinze et al. (2008), there exist students who understand only either hypothetical syllogism or universal instantiations. As such, we argue that it is necessary to sub-divide the Partial-structural Relational sub-level into (a) universal instantiation and (b) hypothetical syllogism. In other words, related to the two types of deductive reasoning, we can distinguish two aspects of understanding the structure of deductive proofs at the Partial-structural Relational sub-level.

Once a student sees a proof as "whole" (c.f. Yang and Lin, 2008, p. 63; the fourth process of Heinze et al., 2008) we call this level "Holistic-structural". Here, premises and conclusions are logically connected through universal instantiations and hypothetical syllogism as two intertwined aspects, like the warp and the weft of a textile. After reaching the "Holisticstructural" level, students become able not only to reconstruct previously taught proofs, but are also able to plan and construct their own proofs. In addition, students become aware of the hierarchical structure of the theorems they use and understand the modular structure of their proofs (Mejia-Ramos et al., 2012).

Figure 2 illustrates our overall framework. As we have described, this framework accounts for the local development of the conceptual growth of the structure of deductive proof. This framework can contribute to devising appropriate learning progressions for the teaching and learning of the structure of deductive proofs (see Section 4.2) alongside other conceptual learning. Nevertheless, this framework is not aiming at the long-term developmental process of the structure of proofs from infants to adults. Our framework is local rather than global.

Having given an account of our theoretical framework, an issue of which we are aware is the question of whether actual students' developmental processes would follow this progression. We need to be sensitive to such possibilities in empirical studies of our framework as we turn to showing how we applied our framework to an episode from our classroom research. 


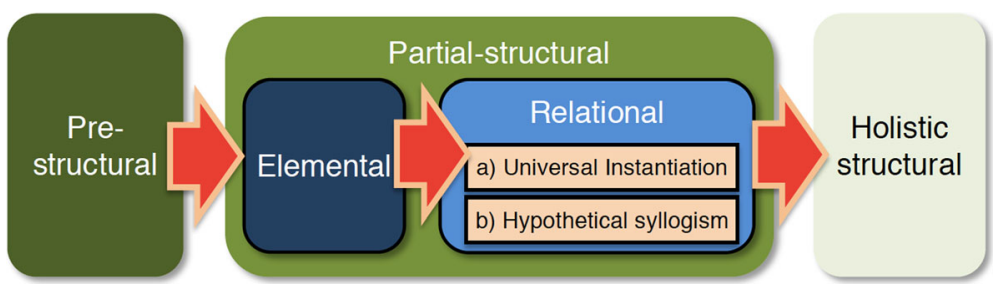

Fig. 2 A framework of understanding of the structure of deductive proofs

\section{Study context and methodology}

\subsection{Students' understanding of deductive proofs in Japanese secondary schools}

In Japan, deductive proof is explicitly taught in "Geometry" in Grade 8. Although there is no official teaching sequence prescribed in the Japanese national "Course of Study", a progression can be found in the seven authorised textbooks and in the practice of many schools (Fujita \& Jones, 2014). Building on informal proofs in earlier grades, students in Grade 8 are introduced to deductive proof through studying properties of angles and lines, triangles, and quadrilaterals; here they learn the structure of deductive proofs and how to construct the proofs, and then explore and prove properties of triangles and properties of quadrilaterals. Recently, proving as an explorative activity (namely planning, improving, and advancing a proof, in addition to its construction) is also stressed in teaching and assessment (Miyazaki \& Fujita, 2015). The geometrical statements studied in Grade 8 are "locally organised" (Freudenthal, 1971) mainly via parallel lines and congruent triangles. Within this "system" (in the sense of 'Mathematical Theorem'; see Mariotti, 2000), students learn deductive proofs in geometry (Miyakawa, 2016).

As in the case elsewhere in the world, many students in Japan find the learning of deductive proofs very difficult. For example, there is evidence that students who have just started learning the proof may often accept proof with logical circularity (Kunimune, Fujita, \& Jones, 2010).

\subsection{A sequence of lessons to introduce the structure of deductive proofs}

Informed by our theoretical framework, we have undertaken a research project that entailed a series of lessons designed for the teaching of the introductory stages of proof learning for Grade 8 students (aged 14 years old in Japan) in order to enhance their capabilities with deductive proof in geometry. By Grade 8 , students are given learning opportunities to explain why geometrical properties are correct, and to deduce them by using already-learned properties. The experimental lessons we designed aimed to initiate Japanese students inexperienced in proof into mastering deductive proofs fruitfully so that they could develop the competencies to construct such proofs for themselves and tackle the more-complicated proofs encountered in subsequent lessons.

One of the features of the sequence of lessons that we designed is the use of flow-chart proofs (see, for example, McMurray, 1976), a format that pictures the deductive relations amongst singular and universal propositions from premises to conclusion, as per the example in Fig. 3. The idea is that a flow-chart proof can be used before using other proof formats such as paragraph proofs or two-column proofs. Related ideas can be found in Gardiner (2004), in 


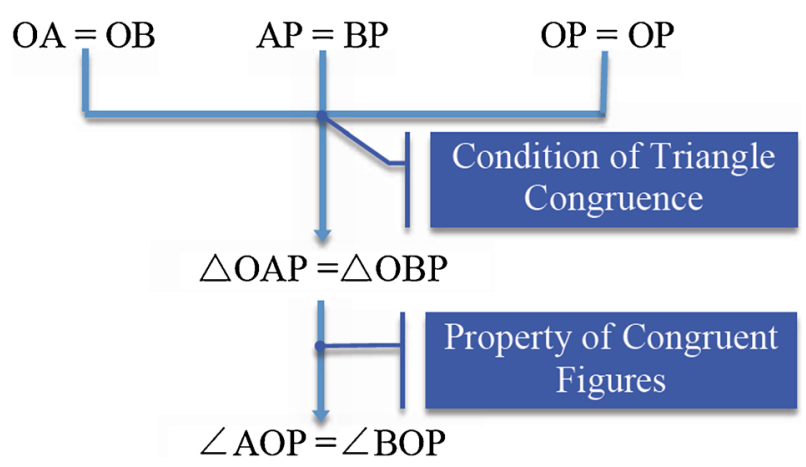

Fig. 3 An example of a flow-chart proof

Leron (1983), and in Back, Mannila, and Wallin (2010). Furthermore, the representational characteristic of flow-chart proof can take the role of mediational means between natural language and functional language (Balacheff, 1987) as symbolic systems. Accordingly, flowchart proofs as "transitional auxiliary representation" (Duval, 2006, p. 111) would help provide a visualisation of both the connection between singular propositions via hypothetical syllogism and the connections between a singular proposition and the necessary universal proposition in the form of universal instantiation towards the Partial-structural Relational sublevel of the structure of deductive proofs.

The design of the lessons had three learning phases as follows: (1) constructing flow-chart proofs in an open situation (four lessons); (2) constructing a paragraph proof by reference to a flow-chart proof in a closed situation (three lessons); and (3) refining paragraph proofs by placing them into a flow-chart proof format in a closed situation (two lessons). Here, an open situation is a proof problem for which there is more than one acceptable proof, while a closed situation is a proof problem for which there is only one acceptable proof.

In the first learning phase, constructing flow-chart proofs in an open problem situation helped to scaffold students' understanding of the structure of deductive proofs (Miyazaki, Fujita, \& Jones, 2015). Since students at this early stage of learning about a deductive proof might see it as a rather meaningless set of symbols about the properties of geometric shapes, through the follow-chart proving we provided a visualisation of the structure of the proofs. By this graphical representation, this phase was designed to support students to understand how to "assemble" a proof as a structural entity. We expected this to support students' shifts of their levels from Partial-structural to Holistic level.

We illustrate the tasks by the example in Fig. 4 intentionally designed so that the premises used to draw a conclusion can be chosen freely. Here, students are likely first to consider which properties and theorems should be used to prove " $\angle \mathrm{B}=\angle \mathrm{D}$ " (i.e. thinking backward), then how premises and conclusions should be deductively organised, which theorems should be used (i.e., thinking forward), and so on. In our design, such a task aims to stimulate flexible forward/backward approaches to proof construction (Matsuda \& VanLehn, 2005). Thus, we can expect that flow-chart proofs in an open situation encourage thinking forward/backward interactively, accompanied by relational understanding of the structure of deductive proofs.

In the second learning phase, during which the use of open problems is reduced, the learning was designed to shift from graphical flow-chart format to paragraph format. Here students were expected to construct flow-chart proofs in a "closed" situation (a problem with the proposed remises/“givens" and conclusions, as typically appear in textbooks; e.g. a 
In the following diagrams, we would like to prove $\angle \mathrm{B}=\angle \mathrm{D}$. What do we need to show this, and what conditions of congruent triangles can be used? Complete the flow-chart !

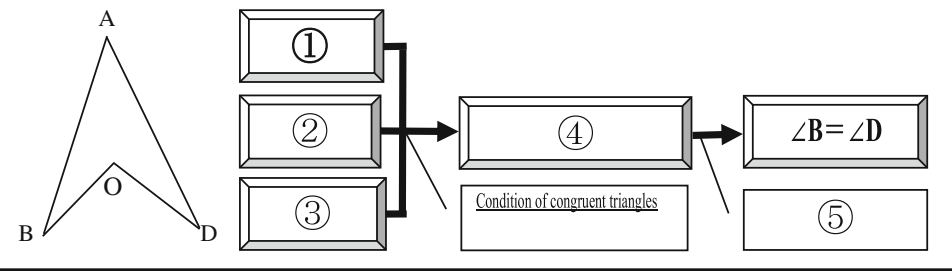

Fig. 4 A flow-chart proof in open situation provided for students

problem as in Fig. 1). Given that during the first phase students would have become familiar with constructing the "storyline" of a proof, in this second phase they especially learnt how to translate the arrows in the flow-chart proofs corresponding to hypothetical syllogism into the appropriate linguistic tokens.

Finally, in the third learning phase, students constructed paragraph proofs in closed problem situations from scratch, and then refined their proofs by placing them into flow-chart proof format if necessary.

\subsection{Data analysis procedure}

For the purposes of this paper, we select episodes from the lessons from the three phases implemented in 2008 in a class of Mr. Y, a teacher with more than 20 years of experiences of mathematics teaching. In this Grade 8 class in a state school in Japan, there were 21 boys and 9 girls, all considered as "typical" for Grade 8 . For our research, all lessons were video-recorded, with this data supplemented by field notes.

After preliminary analysis, we particularly noted the first and fourth lessons in the first phase were the most interesting, as students' Pre- and Partial-structural elemental sub-level of understanding was particularly observable. In the first lesson, the students had used a one-step flow-chart proof to prove that two given triangles were congruent, but in the fourth lesson they tackled the proof problem in Fig. 4 which consists of two steps of deductive reasoning: deducing the congruency of triangles from the assumptions and deducing the equivalence of angles from the triangle congruency. The two lessons were transcribed in full and were analysed qualitatively in terms of the framework presented in Section 3.

\section{Analysis of classroom episodes}

\subsection{Pre-structural level and partial-structural elemental sub-level of understandings}

One example of pre- and partial-structural elemental sub-levels of understandings occurred in the first lesson of the first phase when the students, who had just started learning about deductive proofs with congruent triangles, undertook the multiple-solution problem illustrated in Fig. 5.

In the lesson, a student tried to use "AO $=\angle B D O "$ as one of the premises. This can be considered as Pre-structural level because it is a rather random attempt to use a geometric fact. Another student thought of using the SSS condition of congruency to complete a proof but 
In the diagram below, we know $\mathrm{AO}=\mathrm{BO}$. We want to make $\triangle \mathrm{ACO}$ and $\triangle \mathrm{BDO}$ congruent. Which angles and sides should be equal and what condition of congruent triangles should be used? Complete the flow-chart!

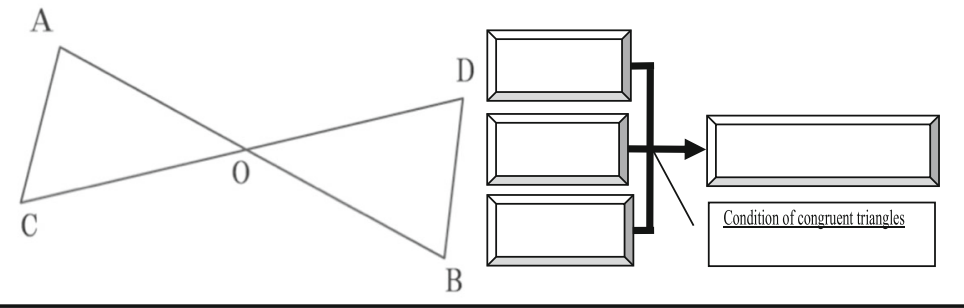

Fig. 5 A congruent triangles problem for Grade 8 students

could not specify which pairs of sides should be chosen. Later this student tried to use " $\angle \mathrm{AOC}=\angle \mathrm{BOD}$ " despite the SSS condition. This is an example of a Partial-structural Elemental sub-level response as the student was starting to pay attention to some components of proof, but did not know how to specify these components in accordance with the SSS theorem chosen (i.e. universal instantiation).

Mr Y then gave this student a hint; he said "In this diagram, which corresponding sides should be chosen to use the condition 'If corresponding three sides are equal, then the two triangles are congruent'?". At this point, the student said "OK, now I understand what I have to do...", and wrote " $\mathrm{CO}=\mathrm{DO}, \mathrm{DB}=\mathrm{AC}$ ". This response can be considered as an illustration of the early stage of the next level "Partial-Structural Relational" as the student could deduce a singular proposition "If $\mathrm{AO}=\mathrm{BO}, \mathrm{CO}=\mathrm{DO}, \mathrm{AC}=\mathrm{DB}$ then $\triangle \mathrm{ABD} \equiv \triangle \mathrm{ACD}$ " is deduced from triangle a congruent theorem (a universal proposition). Nevertheless, it is unclear whether this student would be able to understand the relation between universal and singular propositions in general or whether their understanding remained limited to the case of congruent triangles.

\subsection{Partial-structural relational sub-level of understanding}

\subsubsection{Understanding of syllogism but not universal instantiation}

In the fourth lesson of the first phase, Mr Y posed the problem in Fig. 4 and asked students "What do we need to have in order to prove $\angle \mathrm{B}=\angle \mathrm{C}$ ?". The students worked on this problem and, based on their ideas, agreed that a way to reach a solution would be to draw a line AO and then show two congruent triangles $\triangle \mathrm{ABO} \equiv \triangle \mathrm{ADO}$. All of them constructed more than three suitable proofs using the use of flow-chart format.

Most students could complete the boxes of singular proposition in the flow-chart format, but some students could not answer the box which requests the property of congruent figures (the boxes 4 and 5 in the flow-chart proof of Fig. 4) despite the boxes concerning singular propositions being completed. For example, student $\mathrm{M}$ could answer correctly the boxes of singular propositions as follows: $\mathrm{OA}=\mathrm{OA}, \mathrm{BO}=\mathrm{DO}, \mathrm{AB}=\mathrm{AD}$, but he left the box 4 blank and wrote: "Because $\triangle \mathrm{ABO}$ and $\triangle \mathrm{ADO}$ are congruent" in box 5 rather than using the property of congruent figures that "in congruent triangles all corresponding interior angles are equal". Additionally, despite comparing his incorrect answer with the correct ones of his classmates, he did not notice his error nor try to correct it. If this student fully understood universal 
instantiation, then we would have expected that he could distinguish singular propositions and a universal proposition and tried to express the universal one with the general words not including the symbols of the figure at least, which he could not do. As such, from the point of view of the structure of deductive proofs, this indicates that student $\mathrm{M}$ did not notice that he should show the theorem necessary to deduce $\angle \mathrm{B}=\angle \mathrm{D}$ from $\triangle \mathrm{ABO} \equiv \triangle \mathrm{ADO}$ although he could connect premise $\mathrm{OA}=\mathrm{OA}, \mathrm{BO}=\mathrm{DO}, \mathrm{AB}=\mathrm{AD}$ and conclusion $\angle \mathrm{B}=\angle \mathrm{D}$ by reasoning deductively with the use of hypothetical syllogism.

\subsubsection{Understanding of universal instantiation but not syllogism}

In the same lesson, after individual student working, Mr Y chose to display five students' different proofs on the blackboard. Of the five proofs, three were correct; the other two used the conclusion " $\angle \mathrm{B}=\angle \mathrm{D}$ " as a premise - namely, the solutions fell into logical circularity. $\mathrm{Mr}$ $Y$ checked whether each proof was correct - or not - during the classroom discussion. At this point, some students noticed that " $\angle \mathrm{B}=\angle \mathrm{D}$ " was used in both the premise and conclusion boxes in a proof by student A (Fig. 6).

Even though in the previous lessons student A managed to construct correct proofs using onestep reasoning concerning triangle congruency (as in Fig. 5), in this lesson of two steps of reasoning, student A constructed four flow-chart proofs, the first three of which were entirely correct as she properly could decide the premises. Only one of her proofs, shown in Fig. 6, fell into logical circularity. According to her correct proofs, she could understand which theorems should be used to deduce a singular proposition from a universal proposition (i.e., universal instantiation) although she might not have made it clear what should be considered as premises.

After checking the proofs on the blackboard (see the photograph in Fig. 6), the teacher led a discussion about student A's proof that used $\angle B=\angle D$ in both the premise and conclusion
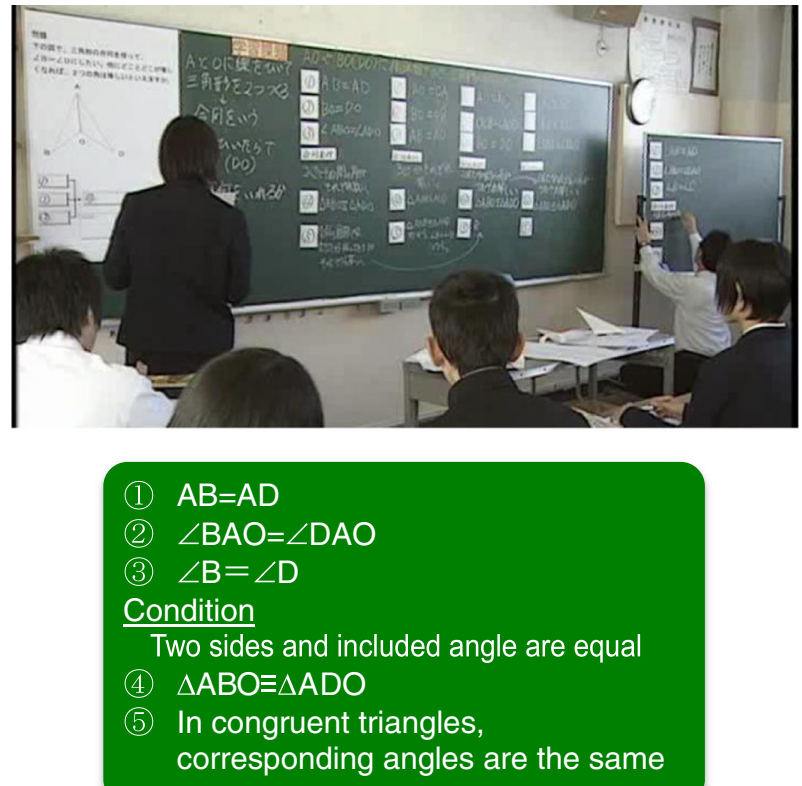

Fig. 6 Proof by student A 
boxes. In this discussion, student $\mathrm{K}$, for example, considered that it would not be appropriate to use " $\angle \mathrm{B}=\angle \mathrm{D}$ " in a proof as "We do not have to write ' $\angle \mathrm{B}=\angle \mathrm{D}$ ' in box 3 like you [student A] because this is already there in the proof [on the board]" (transcript line 283: K). Another student, $\mathrm{S}$, also pointed out this flaw “" $\angle \mathrm{B}=\angle \mathrm{D}$ ' is the answer to the question, at the end of our flow-chart, umm, and if we use it in our proof, then, we have already said they were equal? So, there is no point to write it again if we have already said this?" (transcript line 300: S). Even so, student A defended her proof as "I meant ' $\angle \mathrm{B}=\angle \mathrm{D}$ ' is a premise in my proof, and I used premises to prove two triangles are congruent, and then I reached the conclusion, so I think it is OK" (transcript line 311: A).

Then the following conversation took place amongst the three students:

\section{Student S I think, if we use a premise it would be something we assume is true, and if it is true, then isn't it already an answer? I think it is pointless [to use ' $\angle B=\angle D$ ' in a proof] \\ 323 Student A W, why is it pointless? \\ 324 Teacher I think [student K] wants to add something? Please, [student K]. \\ 325 Student $\mathrm{K}$ I think in this problem we have to prove ' $\angle \mathrm{B}=\angle \mathrm{D}$ ', or we want to prove this will be true, and, I just thought we would not be able to use this there [in the box 3], I just wanted to say this.}

326 Student A I think we can use it (' $\angle \mathrm{B}=\angle \mathrm{D}$ ')!

Student $\mathrm{S}$ and student $\mathrm{K}$ considered "already true statements" can be used as the premises, and if " $\angle B=\angle D$ " is used as a premise, then this means " $\angle B=\angle D$ " is already true before being proved. Moreover student $\mathrm{K}$ sharply pointed out that using " $\angle \mathrm{B}=\angle \mathrm{D}$ " as a premise led them to tautology (transcript line 325), but student A could not be convinced due to without recognising the appearance of tautology. This is an illustration that there is a case that student A might understand universal instantiation but not syllogism. In other words, that student A accepted logical circularity can be an indicator of a lack of understanding of syllogism.

\section{Discussion}

\subsection{Two independent aspects of understanding: universal instantiations and hypothetical syllogism}

In our theoretical framework of students' understanding of the structure of deductive proofs with three levels: Pre-structural, Partial-structural, and Holistic-structural, we consider that understandings concerning universal instantiations and hypothetical syllogism are independent, and the classroom evidence that we have provided suggests this is the case. In the first episode (Section 5.2.1), some students who could logically connect the elements of proofs could not state clearly which theorems should be used to ground the reason. In the second episode (Section 5.2.2), the students could complete each box in the flow-chart proof and could state correctly which triangles were congruent by using appropriate theorems (a use of universal propositions). From these facts, their levels of understanding are at least at the Partial-structural Relational sub-level, yet with only partial understanding of universal instantiation. Thus, the necessity of sub-division of Partial-structural Relational sub-level into (a) universal instantiation and (b) hypothetical syllogism has been empirically justified. What is 
more, some students, such as student S (transcript line 322: S) and student K (transcript line 325: K), could point out the roles of premises and they judged that it would not be appropriate to use " $\angle \mathrm{B}=\angle \mathrm{D}$ " as a premise to prove the conclusion " $\angle \mathrm{B}=\angle \mathrm{D}$ ". This is indicative of their understanding being at the beginning of the Holistic level.

\subsection{Acceptance of logical circularity as indicator of lack of understanding of syllogism}

We showed how student $A$, for example, considered that a premise is necessary to prove a statement locally, and she just used " $\angle \mathrm{B}=\angle \mathrm{D}$ " as a premise for her proof. We also noted that she understood that the singular proposition " $\angle \mathrm{B}=\angle \mathrm{D}$ " is the conclusion. In addition, student $\mathrm{A}$ insisted that " $\angle \mathrm{B}=\angle \mathrm{D}$ " can be used to prove " $\angle \mathrm{B}=\angle \mathrm{D}$ " (transcript lines 311: A; 326: A). She explained her reasoning as " $\angle \mathrm{B}=\angle \mathrm{D}$ " can be used to deduce two triangles are congruent, and from congruent triangles it can be deduced " $\angle \mathrm{B}=\angle \mathrm{D}$ "; therefore, it would be no problem for her to use this in her proof. We consider that this viewpoint was her lack of understanding of hypothetical syllogism that connects singular propositions in a proof. She could see the following two parts of her proof were logically true: (specifically, $1 . \mathrm{AB}=\mathrm{AD}, 2 . \angle \mathrm{BAO}=\angle \mathrm{DAO}, 3 . \angle \mathrm{B}=\angle \mathrm{D}$, Fig. 7 below upper left) and (4. $\triangle \mathrm{ABO} \equiv \triangle \mathrm{ACO}$ and $5 . \triangle \mathrm{ABO} \equiv \triangle \mathrm{ACO} \rightarrow \angle \mathrm{B}=\angle \mathrm{D}$, Fig. 7 upper right). Each part includes a singular proposition of these components that was universally specified from theorems.

While this is not problematic when these parts are examined individually, to complete a proof, these two parts (left and right) should be connected by hypothetical syllogism as shown in Fig. 7. Yet in the case of student A this is impossible because the statement to be concluded is used as an assumption, i.e., there is circular reasoning. As our data show, some students in our study had no problem to accept this as a valid proof, because, we argue, they see that if each part of a proof is logically true, and the connection of these parts is possible, then the whole proof would be necessarily true. They could not see a proof as "whole" in which premises and conclusions are logically connected by hypothetical syllogism.

By considering students like A, we consider that this phenomenon of accepting logical circularity is strongly related to their lack of understanding of hypothetical syllogism. Conversely, rejecting such a proof can be used as in indicator for judging that these students might have reached, or be reaching, Partial-structural Relational level (b) hypothetical syllogism.

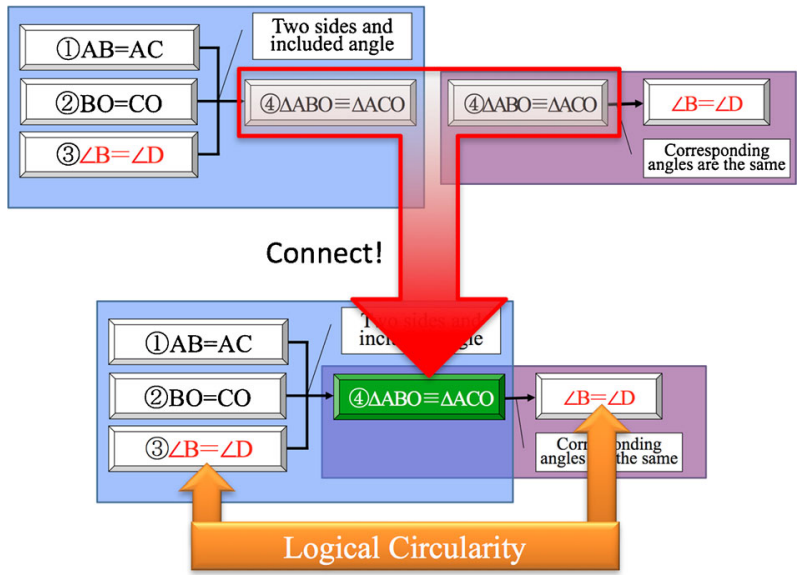

Fig. 7 Representation of student A's understanding of her proof with logical circularity 


\section{Conclusion}

The teaching and learning of deductive proofs in mathematics is one of the most important goals in mathematics education. The framework presented in this paper, in focussing on students' developing understanding of the structure of deductive proofs, is offered as a potentially valuable model to improve the teaching and learning of proof in mathematics. While related studies (e.g., the work on proof schemes, Harel \& Sowder, 2007) have revealed how students perceive a proof, our framework has a possibility to identify other causes of difficulties of proof in the introductory stage of learning to construct deductive proofs (around Level 3 and 4 of van Hiele's levels) and in the bridging process of their proving (Heinze et al., 2008). At this stage of learning, students can find it difficult to identify which theorem (or theorems) to use to deduce intermediate statements or a conclusion, or they can accept a proof even when it contains logical circularity. As our classroom evidence indicates, such students are still at the Partial-structural Relational sub-level as they lack the ideas of universal instantiation and/or hypothetical syllogism. Existing studies have not fully explained this. While we have not so far found different routes of progression to the one we suggest in our framework (see Fig. 2), investigating this issue further is one of our future tasks.

Our framework offers insights that could inform curriculum design and instructional development for effective teaching of deductive proofs. In designing an effective curriculum for deductive proof in secondary school, we argue that it would help if the levels of understanding of the structure of deductive proof were taken into consideration, with special attention given to two independent aspects of Relational understanding: universal instantiations and hypothetical syllogism. To develop instructional approaches, we argue that even after "telling" students what is assumed and what is proved in a proof, it remains uncertain what the students consider about circular reasoning and hence they may continue to accept proofs with logical circularity. Meanwhile, we consider a proof with logical circularity provides a good opportunity for students to consider what a proof is, what roles premises have, how the two kinds of deductive reasoning work, and so on. Future research might focus on how teachers can encourage learners to focus on the structural relationships between premises and conclusion and how these can be bridged through forms of hypothetical syllogism.

Acknowledgments This research was supported by grants from the Japanese Ministry of Education, Culture, Sports, Science, and Technology (JP24243077, JP26282039, JP16H03057, JP16H02068), and The Daiwa Anglo-Japanese foundation.

Open Access This article is distributed under the terms of the Creative Commons Attribution 4.0 International License (http://creativecommons.org/licenses/by/4.0/), which permits unrestricted use, distribution, and reproduction in any medium, provided you give appropriate credit to the original author(s) and the source, provide a link to the Creative Commons license, and indicate if changes were made.

\section{References}

Back, R. J., Mannila, L., \& Wallin, S. (2010). 'It takes me longer, but I understand better': Student feedback on structured derivations. International Journal of Mathematical Education in Science and Technology, 41(5), $575-593$.

Balacheff, N. (1987). Processus de preuve et situations de validation. Educational Studies in Mathematics, 18(2), $147-176$.

Baum, L. A., Danovitch, J. H., \& Keil, F. C. (2008). Children's sensitivity to circular explanations. Journal of Experimental Child Psychology, 100(2), 146-155. 
de Villiers, M. D. (1990). The role and function of proof in mathematics. Pythagoras, 24, 17-24.

Durand-Guerrier, V., Boero, P., Douek, N., Epp, S. S., \& Tanguay, D. (2012). Examining the role of logic in teaching proof. In G. Hanna \& M. de Villiers (Eds.), Proof and proving in mathematics education: The ICMI Study (pp. 369-389). New York: Springer.

Duval, R. (2002). Proof understanding in mathematics: What ways for students? In F. L. Lin (Ed.), Proceedings of the international conference on mathematics: Understanding proving and proving to understand (pp. 6177). Taipei: National Taiwan Normal University.

Duval, R. (2006). A cognitive analysis of problems of comprehension in a learning of mathematics. Educational Studies in Mathematics, 61(1-2), 103-131.

Empson, S. B. (2011). On the idea of learning trajectories: Promises and pitfalls. The Mathematics Enthusiast, $8(3), 571-596$.

Fawcett, H. P. (1938). The nature of proof. New York: AMS Press.

Freudenthal, H. (1971). Geometry between the devil and the deep sea. Educational Studies in Mathematics, 3(34), 413-435.

Fujita, T., \& Jones, K. (2014). Reasoning-and-proving in geometry in school mathematics textbooks in Japan. International Journal of Educational Research, 64, 81-91.

Gardiner, T. (2004). Learning to prove: Using structured templates for multi-step calculations as an introduction to local deduction. ZDM: International Journal on Mathematics Education, 36(2), 67-76.

Gray, E., \& Tall, D. O. (1994). Duality, ambiguity and flexibility: A proceptual view of simple arithmetic. Journal for Research in Mathematics Education, 26(2), 115-141.

Hanna, G., \& de Villiers, M. (2008). ICMI Study 19: Proof and proving in mathematics education. ZDM: International Journal on Mathematics Education, 40(2), 329-336.

Hanna, G., \& de Villiers, M. (2012). Aspects of proof in mathematics education. In G. Hanna \& M. de Villiers (Eds.), Proof and proving in mathematics education: The ICMI Study (pp. 1-10). New York: Springer.

Hanna, G., \& Jahnke, H. N. (1993). Proof and application. Educational Studies in Mathematics, 24(4), 421-439.

Harel, G., \& Sowder, L. (2007). Toward a comprehensive perspective on proof. In F. Lester (Ed.), Second handbook of research on mathematics teaching and learning (pp. 805-842). Reston: VA: NCTM.

Heinze, A., Cheng, Y. H., Ufer, S., Lin, F. L., \& Reiss, K. (2008). Strategies to foster students' competencies in constructing multi-steps geometric proofs: Teaching experiments in Taiwan and Germany. ZDM: International Journal on Mathematics Education, 40(3), 443-453.

Herbst, P. G., \& Brach, C. (2006). Proving and doing proofs in high school geometry classes: What is it that is going on for students? Cognition and Instruction, 24(1), 73-122.

Hoyles, C., \& Healy, L. (2007). Curriculum change and geometrical reasoning. In P. Boero (Ed.), Theorems in school (pp. 81-115). Rotterdam: Sense.

Inglis, M., \& Alcock, L. (2012). Expert and novice approaches to reading mathematical proofs. Journal for Research in Mathematics Education, 43(4), 358-390.

Küchemann, D., \& Hoyles, C. (2006). Influences on students' mathematical reasoning and patterns in its development: Insights from a longitudinal study with particular reference to geometry. International Journal of Science and Mathematics Education, 4(4), 581-608.

Kunimune, S., Fujita, T., \& Jones, K. (2010). Strengthening students' understanding of 'proof' in geometry in lower secondary school. In V. Durand-Guerrier, S. Soury-Lavergne and F. Arzarello (Eds), Proc. 6th Congress of the European Society for Research in Mathematics Education (pp. 756-765). Lyon, France: ERME.

Leron, U. (1983). Structuring mathematical proofs. American Mathematical Monthly, 90(3), 174-185.

Lin, F., \& Yang, K. (2007). The reading comprehension of geometric proofs: The contribution of knowledge and reasoning. International Journal of Science and Mathematics Education, 5(4), 729-754.

Mariotti, M. A. (2000). Introduction of proof: The mediation of a dynamic software environment. Educational Studies in Mathematics, 44, 25-53.

Martinez, M. V., \& Pedemonte, B. (2014). Relationship between inductive arithmetic argumentation and deductive algebraic proof. Educational Studies in Mathematics, 86(1), 125-149.

Matsuda, N., \& VanLehn, K. (2005). Advanced Geometry Tutor: An intelligent tutor that teaches proof-writing with construction. In C.-K. Looi, G. I. McCalla, B. Bredeweg, \& J. Breuker (Eds.), Proceedings of the 12th International Conference on Artificial Intelligence in Education (pp. 443-450). Amsterdam: IOS Press.

McCrone, S. S., \& Martin, T. S. (2004). Assessing high school students' understanding of geometric proof. Canadian Journal for Science, Mathematics, and Technology Education, 4(2), 223-242.

McMurray, R. (1976). Geometry: A flow proof approach. Mathematics Teacher, 71, 592-595.

Mejia-Ramos, J. P., Fuller, E., Weber, K., Rhoads, K., \& Samkoff, A. (2012). An assessment model for proof comprehension in undergraduate mathematics. Educational Studies in Mathematics, 79(1), 3-18.

MEXT. (2014). Reports of National Assessment of Academic Ability: Mathematics. Retrieved from http://www. nier.go.jp/14chousakekkahoukoku/report/data/mmath_05.pdf (in Japanese). 
Miyakawa, T. (2016). Comparative analysis on the nature of proof to be taught in geometry: The cases of French and Japanese lower secondary schools. Educational Studies in Mathematics, 1-18. doi: 10.1007/s10649016-9711-x

Miyazaki, M., \& Fujita, T. (2015). Proving as an explorative activity in mathematics education: New trends in Japanese research into proof. In B. Sriraman et al. (Eds.), First sourcebook on Asian research in mathematics education: China, Korea, Singapore, Japan, Malaysia and India (pp. 1375-1407). Charlotte: Information Age Publishing.

Miyazaki, M., \& Yumoto, T. (2009). Teaching and learning a proof as an object in lower secondary school mathematics of Japan. In F.-L. Lin, F.-J. Hsieh, G. Hanna, \& M. de Villiers (Eds.), Proceedings of ICMI Study 19 conference, proof and proving in mathematics education (Vol. 2, pp. 76-81). Taiwan: National Taiwan Normal University.

Miyazaki, M., Fujita, T., \& Jones, K. (2015). Flow-chart proofs with open problems as scaffolds for learning about geometrical proofs. ZDM: International Journal on Mathematics Education, 47(7), 1-14.

Pegg, J., \& Tall, D. (2010). The fundamental cycle of concept construction underlying various theoretical frameworks. In B. Sriraman \& L. English (Eds.), Theories of mathematics education: Seeking new frontiers (pp. 173-192). New York: Springer.

Rips, L. J. (2002). Circular reasoning. Cognitive Science, 26, 767-795.

Senk, S. L. (1989). Van Hiele levels and achievement in writing geometry proofs. Journal for Research in Mathematics Education, 20(3), 309-321.

Stylianou, D. A., Blanton, M. L., \& Rotou, O. (2015). Undergraduate students' understanding of proof: Relationships between proof conceptions, beliefs, and classroom experiences with learning proof. International Journal of Research in Undergraduate Mathematics Education, 1(1), 91-134.

Usiskin, Z. (1982). Van Hiele levels and achievement in secondary school geometry. Chicago: University of Chicago.

van Hiele, P. M. (1959/1984). A child's thought and geometry. In D. Fuys, D. Geddes and R. W. Tischler (Eds.) English translation of selected writings of Dina van Hiele-Geldof and Pierre M. van Hiele (pp.243-252). Washington, D.C.: NSF.

Weber, K. (2001). Student difficulty in constructing proofs: The need for strategic knowledge. Educational Studies in Mathematics, 48(1), 101-119.

Weber, K., \& Alcock, L. (2005). Using warranted implications to understand and validate proofs. For the Learning of Mathematic, 25(1), 34-38.

Yang, K. L., \& Lin, F. L. (2008). A model of reading comprehension of geometry proof. Educational Studies in Mathematics, 67(1), 59-76. 